\title{
Clinical Comparative Analysis of Neonatal Scalp Vein and Axillary Vein Catheterization
}

H Li, F Liu, W Zhang, B Zhao, Y Wang, P Wang

\begin{abstract}
Objective: The aim of the current study was to study the application effects of axillary vein catheterization

(AVC) in the clinical nursing.

Methods: A retrospective analysis was carried out towards the detailed information of children that were performed AVC, and the related situations of scalp vein catheterization (SVC) and AVC were compared.

Results: The birth weight, gestational age, gender ratio, age when catheterized, catheterization duration and liquid infusion time had no statistically significant difference; the rate of catheter ectopia, phlebitis and leaking of SVC were significantly higher than those of AVC, and the differences were statistically significant.
\end{abstract}

Conclusions: AVC was safe and effective towards the neonates, especially in the rescue and care of critically ill newborns, the axillary vein catheter infusion was safe and had fewer complications, which could reduce the pain caused by the repeated puncture, improve the nurses' work efficiency, and ensure safe and timely infusion of intravenous nutrition fluid, so it could be used as the preferred choice for the neonatal intensive care unit (NICU) neonatal intravenous remaining, thus it was worthy of the neonatal clinical application.

Keywords: Axillary vein, catheterization, clinical comparative analysis, newborns, scalp vein

From: Division of Paediatrics Department, the Central Hospital of Xinxiang, Xinxiang 453000, China.

Correspondence: Dr H Li, Division of Paediatrics Department, the Central Hospital of Xinxiang, No 56 Jinsui Road Weibin District, Xinxiang 45300, China. Fax: +86 373 2048931, e-mail: haixialicn@163.com 


\section{INTRODUCTION}

Clinically, some newborn children needed the intravenous infusion of nutritious solution, as well as certain stimulant medication, for a very long time (1). Due to the congenital shortages of neonatal peripheral vessels, the leakage would easily occur, thus leading to such care complications as phlebitis, skin necrosis, infection and others (2). In recent years, scalp vein catheterization (SVC) reduced the workload of nurses, especially towards the critically ill children, it could facilitate the rescue, save the time, and improve the salvage rate (3). SVC had gradually become a common infusion way in the pediatrics, which better solves the problems of fluid replacement and medication, while it also existed the complications as puncture failure, phlebitis and indwelling needle abscission, etc (4). And it was found in the practice that it would be affected by multiple factors, and prone to phlebitis (5). Although as the replacement product of SVC and had many advantages, in fact, the problems caused by intravenous catheterization still needed some thinking (6).

Towards the above problems, this paper proposed axillary vein catheterization (AVC) technology, and compared it with SVC to improve the effects of neonatal intravenous catheterization and reduce the complications, AVC infusion was safe (7). As the rescue infusion pathway towards the critically ill newborns, compared with the catheterization in other body parts, AVC was superior in the puncture success rate, retention time and complication care (8). The investigation of AVC in neonates was important, the adverse reactions were less, and the minor-lateral position, with the arm outer-extending degree as $110-145^{\circ}$, had high puncture success rate (9). When rescuing the critically ill newborns, AVC infusion was safe, and the complications were less, thus it could be used as the preferred 
intravenous catheterization choice towards the NICU newborns. thus it was worthy of the clinical application.

\section{MATERIALS AND METHODS}

In the clinical care, the neonatal intravenous infusion was an important way of clinical administration, as well as an important nursing technique (10). The intravenous indwelling needle, also known as the trocar, was made of the advanced biological materials, applied into the clinics as the replacement of scalp acupuncture in 1958, and widely used in the United States and Europe 30 years ago (11). Clinically, most neonatal patients were used the scalp vein and limb superficial vein for the puncturing catheterization, which was prone to occur the leakage and phlebitis, especially in the infusion of hypertonic liquid and vasoactive drugs, the stimuli to the blood vessels were huge (12). Our division applied the vein catheterization towards 136 newborns in neonatal intensive care unit (NICU) of our hospital from June 2012 to June 2013, among who 13 cases of treatment abandonment were excluded, and 8 cases died, the rest 115 cases were discharged after the recovery, so they were enrolled into this study. The detailed information of the child patients were retrospectively analyzed, aiming to compare the relevant situations occurred during the scalp and axillary vein catheterization.

\section{Subjects}

Our department performed 136 cases of vein catheterization towards the NICU newborns from June 2012 to June 2013, after excluded 13 cases of treatment abandonment, 8 cases of 
death, the rest 115 cases were discharged after the treatment, and they were included into this study. The detailed information of these children was retrospectively analyzed to compare the related situations of SVC and AVC. This study was conducted in accordance with the declaration of Helsinki. This study was conducted with approval from the Ethics Committee of the Central Hospital of Xinxiang. Written informed consent was obtained from all participants' guardians.

\section{SVC}

The skilled nurses operated the vein catheterization. The needle used the disposable Intima11 intravenous needle (Becton Dickinson Medical Devices Co., Ltd. Suzhou), according to the distributions of blood vessels, the puncture sites chose such thicker vessels as posterior auricular vein, superficial temporal vein, frontal median vein and frontal horn branch. Firstly, the hair at and around the blood vessel was shaved for about $6 \mathrm{~cm}$, then $0.25 \%$ povidoneiodine was used to disinfect the skin puncture site, followed by the $75 \%$ alcohol deiodination and air-dryign. During the puncture, the heparin cap was inserted into the scalp vein to drain the air, so that the adhesions between the casing and needle core could be prevented, then closed the regulator. The right hand hold the needle wing, and formed $15-25^{\circ}$ angle towards the scalp, then inserted the needle, the speed should be slow and the blood return should be observed. When the needle core was withdrawn, the outer tube was inserted, until it was all placed into the tube, after observed that the infusion was smooth and had no extravasation, the needle could be fixed with a transparent applicator, with injection time stated on the paper tape, and the extension tube fixed on the child's head. 


\section{AVC}

The catheterization was operated by the same skilled nurse, the needle was the same, while the patient was in the supine position, with one arm straightened outwards to fully expose the armpit. The axillary vein connected the end of subclavian vein, and the site where it intersected with the clavicle was about $65.7+6.2 \mathrm{~mm}$ away from the collarbone inner end (13), and started from the intersection of clavicular 2/5 inner side and 3/5 outer side, the diameter was $12.3+0.2 \mathrm{~mm}$, it located inside the axillary artery, when touched the pulsatility of axillary artery, the needle should be inserted under the armpit $0.3 \mathrm{~cm}$ inner from the site where the most obvious beat was felt, and when the pulsatility was unclear, the needle was inserted at the intersection of $0.2-0.4 \mathrm{~cm}$ to the armpit midpoint and 1.0-1.2 $\mathrm{cm}$ under (14). The conventional disinfection was then performed towards the puncture site, the left hand of the operator held the upper arm of the child to tense the lower skin, then slowly inserted the needle $0.5-1 \mathrm{~cm}$ beneath the puncture point with the angle as $10-15^{\circ}$. When the blood return was seen, the needle was parallel inserted $0.1-0.2 \mathrm{~cm}$, and withdrew the needle core, the soft core was then sent into the axillary vein, to ensure the indwelling needle kept inside the vessel, the saline syringe should be connected, when the blood could be withdrawn smoothly, the saline was injected to seal the tube, and $3 \mathrm{M}$ transparent applicator was used for the fixation.

\section{Post-catheterization nursing}

The nursing was performed according to conventional venipuncture and extubation nursing, the tube sealing liquid was used for the plugging, and the assessment was performed in 
accordance with the venous catheterization record, the drugs of high nutrition, high sugar (glucose concentration $>12.5 \%$ ) and high osmotic pressure (osmotic pressure $>850 \mathrm{mOsm} / \mathrm{L}$ ) were all infused through the intravenous catheter. The axillary vein was relatively coarse, close to the large blood vessel, if the infusion was not smooth, the infusion tube should not be squeezed to prevent the small blood clots forcedly squeezed into the blood circulation and formed the thrombosis (15).

\section{Indwelling time and extubation}

These were up to the decisions of neonate-professional attending physicians and nurses of intravenous infusion group according to the clinical situations. The extubation should be performed immediately when the catheter-related infections and other related complications occurred.

\section{Vein catheterization-related complications}

Catheter-related bloodstream infections (CRBSI): The diagnostic criteria referred to the literature (16), namely the bacterial colonies were cultured from the peripheral blood during the catheterization and within $48 \mathrm{~h}$ after the extubation.

Phlebitis: Mechanical or chemical, not related with infections, exhibited the red streak from the puncture point and along the catheter, and accompanied with the induration.

Catheter blockage: The infusion pump alarmed, the infusion could not continue, and when the catheter was withdrawn, there was no blood return.

Oozing: The fluid exosmosed into the tissue space, causing local swelling and pain. 


\section{Statistical analysis}

The SPSS11.5 software was used for the statistical analysis. The categorical data were expressed by the frequency and rate, the intergroup comparison used the non-parametric test and Fisher exact test; the normally publish measurement data were expressed as mean \pm standard deviation $(\bar{x} \pm s)$, and the intergroup comparison used the two-sample $t$ test, with $P<0.05$ considered as the statistical significance.

\section{RESULTS}

\section{Clinical features}

Among 115 patients, 83 patients were very-low or ultra-low-birth-weight children, and 32 cases were diagnosed as low-birth-weight children, smaller-than-gestational-age children and neonatal hypoglycemia, etc. 61 cases were performed SVC, and 54 cases were performed AVC. The birth weight, gestational age, gender ratio, age when performed the puncture catheterization, catheterization duration and intravenous infusion time had no statistically significant difference (Table 1).

\section{Catheterization-related complications}

The incidence rates of CRBSI of the 2 groups had no statistically significant difference. The pathogens cultured by the blood included Acinetobacter baumannii, Klebsiella pneumoniae, Escherichia coli, Staphylococcus aureus and coagulase-negative staphylococci. The catheter displacement rate, phlebitis and exudation of the SVC group were significantly higher than 
those of the AVC group, and the differences were statistically significant (Table 2).

\section{DISCUSSION}

It could be seen from Table 2, among the 115 cases, the incidence rates of CRBSI of the 2 groups had no statistically significant difference, while the comparisons of catheter displacement rate, phlebitis and exudation exhibited the statistically significant difference. The catheter displacement rate of the SVC group was significantly higher than the AVC group, which was related with that the scalp vein wall was thin, easily flattened, easily slide, the branches were mostly net-like, the return of blood would appear as impulse-like, and the resistance was greater when pushing the medications. While the axillary vein had less branches, thick, straight and easily punctured. So the rate of axillary vein displacement could be significantly lower than the scalp vein. The phlebitis rate of SVC was significantly higher, and this was related with that the lumen diameter of scalp vein was small, with more branches, thus the resistance of fluid infusion was, and the vascular intima would be easily injured, the vascular intima could prevent the liquid leakage. Meanwhile, it would damage the endothelial cells, exposed the subendothelial layer, the stimulation would thus induce the inflammatory reactions, and the endothelial cells could release the negative charges to prevent the platelet from adhering to the vessel wall, thus leading to the formation of thrombosis (17).

Concept change of venipuncture catheterization: people would habitually think that AVC 
was the choice when SVC failed, and because it was located at armpits, sweat or other secretions would easily cause the contamination, thus the risk of infection would be increased. The intravenous infusion was the important way for the neonates to receive the drug therapy and nutritional intake, the practice had proved that the axillary vein puncture could avoid and reduce the stimulation of specific drugs towards the blood vessels, reduce the pain caused by the repeated puncture towards the children (18), so it was suitable for the $24 \mathrm{~h}$ continuous infusion. The osmotic tolerance of axillary vein was similar to that of central vein, so the preterm children that needed the intravenous nutrition could choose the axillary vein infusion, and could receive very good results (19). The results of AVC towards the preterm children were better than the limb-intravenous catheterization, conducive to the clinical medication and rescue, and could reduce the incidence of phlebitis, alleviate the children's suffering, and the drugs could be accurately, fast and safely sent to the therapeutic purpose (20). As it could be seen from this study, AVC did not increase the infection rate, on the contrary, it could increase the ideal place for the indwelling, easy for the fixation, and reduce the displacement rate. Because the postural characteristics of newborns, namely the upper extremity normally extended upwards and exhibited the flexion, and the axillary vein was thick, thus it could avoid the occurrence of phlebitis and oozing, while not easily cause the significant damages and scarring, while SVC would easily occur such care problems as scarring when the exudation and phlebitis occurred, leading to the dissatisfaction, complaints or compensation disputes from the patients' families. Thus the ideas could be changed, and consider AVC as the preferred method. Researched showed that AVC was safe and effective. Compared with SVC, its overall complications were fewer, therefore, AVC could be a better 
choice for the newborns.

\section{CONFLICT OF INTEREST}

All authors have no conflict of interest regarding this paper. 


\section{REFERENCES}

1. Ehrenkranz RA. Ongoing issues in the intensive care for the periviable infantnutritional management and prevention of bronchopulmonary dysplasia and nosocomial infections. Semin Perinatol 2014; 38: 25-30.

2. Wrightson DD. Peripherally inserted central catheter complications in neonates with upper versus lower extremity insertion sites. Adv Neonatal Care 2013; 13: 198-204.

3. Leick-Rude MK, Haney B. Midline catheter use in the intensive care nursery. Neonatal Netw 2006; 25: 189-99.

4. Aggarwal R, Downe L. Use of percutaneous silastic central venous catheters in the management of newborn infants. Indian Pediatr 2001; 38: 889-92.

5. Rastogi S, Bhutada A, Sahni R, Berdon WE, Wung JT. Spontaneous correction of the malpositioned percutaneous central venous line in infants. Pediatr Radiol 1998; 28: 6946.

6. Malbezin S, Gauss T, Smith I, Bruneau B, Mangalsuren N, Diallo T et al. A review of 5434 percutaneous pediatric central venous catheters inserted by anesthesiologists. Paediatr Anaesth 2013; 23: 974-9.

7. Mochida T, Seino Y, Matsuda K, Haga M, Yamamoto G, Moridaira T et al. Safety of axillary and subclavian vein cannulation using real-time ultrasound guidance. Masui 2014; 63: 57-61.

8. Kayashima K, Yoshino H, Ueki M, Kataoka K. On the characteristics of axillary veins and internal jugular veins in pediatric patients. Masui 2011; 60: 1378-83.

9. Sommerkamp SK, Romaniuk VM, Witting MD, Ford DR, Allison MG, Euerle BD. A comparison of longitudinal and transverse approaches to ultrasound-guided axillary vein cannulation. Am J Emerg Med 2013; 31: 478-81.

10. Rangel UV, Gomes Junior SC, Costa AM, Moreira ME. Variables associated with peripherally inserted central catheter related infection in high risk newborn infants. Rev Lat Am Enfermagem 2014; 22: 842-7.

11. Wilson-Storey D. Just a 'wee prick' with a needle. J R Coll Surg Edinb 1996; 41: 412-3.

12. Soong WJ, Jeng MJ, Hwang B. The evaluation of percutaneous central venous catheters-a convenient technique in pediatric patients. Intensive Care Med 1995; 21: 759-65. 
13. Haas NA. Clinical review: vascular access for fluid infusion in children. Crit Care 2004; 8: $478-84$.

14. Pirotte T. Ultrasound-guided vascular access in adults and children: beyond the internal jugular vein puncture. Acta Anaesthesiol Belg 2008; 59: 157-66.

15. Huang ZP, Liu XJ, Zou BX, Wang LG, Zhou T. The complete recanalization of PICCrelated venous thrombosis in cancer patients: a series of case reports. Exp Ther Med 2013; 6: 411-2.

16. O’Grady NP, Alexander M, Burns LA, Dellinger EP, Garland J, Heard SO et al. Guidelines for the preverton of intravascular catheter-related infections. Am J Infect Control 2011; 399: S1-34.

17. Racadio JM, Doellman DA, Johnson ND, Bean JA, Jacobs BR. Pediatric peripherally inserted central catheters: complication rates related to catheter tip location. Pediatrics 2001; 107: E28.

18. Chaturvedi A, Bithal PK, Dash H, Chauhan RS, Mohanty B. Catheter malplacement during central venous cannulation through arm veins in pediatric patients. J Neurosurg Anesthesiol 2003; 15: 170-5.

19. Panagiotounakou P, Antonogeorgos G, Gounari E, Papadakis S, Labadaridis J, Gounaris AK. Peripherally inserted central venous catheters: frequency of complications in premature newborn depends on the insertion site. J Perinatol 2014; 34: 461-3.

20. Tang VC, Morsy MA, Chemla ES. Using arteriovenous fistulae as a dual access for hemodialysis and total parenteral nutrition administration is feasible with a good outcome: a case series. J Vasc Access 2007; 8: 305-8. 
Haixia et al

Table 1. SVC, AVC clinical features of comparison.

\begin{tabular}{|c|c|c|c|c|c|c|c|c|}
\hline \multirow[t]{2}{*}{ Puncture } & \multirow[t]{2}{*}{ Cases } & \multirow{2}{*}{$\begin{array}{l}\text { Birth weight } \\
\qquad(\overline{\mathrm{X}} \pm \mathrm{s}, \mathrm{g})\end{array}$} & \multirow{2}{*}{$\begin{array}{c}\text { Gestational age } \\
(\bar{x} \pm s, W)\end{array}$} & \multicolumn{2}{|c|}{ Baby boy } & \multirow{2}{*}{$\begin{array}{l}\text { Puncture time of age } \\
\qquad(\overline{\mathrm{x}} \pm \mathrm{s}, \mathrm{d})\end{array}$} & \multirow{2}{*}{$\begin{array}{c}\text { Indwelling time } \\
(\overline{\mathrm{x}} \pm \mathrm{s}, \mathrm{d})\end{array}$} & \multirow{2}{*}{$\begin{array}{l}\text { Liquid infusion time } \\
\qquad(\overline{\mathrm{x}} \pm \mathrm{s}, \mathrm{d})\end{array}$} \\
\hline & & & & Cases & PCT $(\%)$ & & & \\
\hline SVC & 61 & $1340 \pm 270$ & $30.5 \pm 2.3$ & 40 & 65.6 & $8.2 \pm 5.8$ & $2.2 \pm 1.2$ & $25.8 \pm 14.3$ \\
\hline AVC & 54 & $1340 \pm 680$ & $29.9 \pm 2.5$ & 29 & 53.7 & $7.5 \pm 6.2$ & $2.5 \pm 1.8$ & $30.2 \pm 19.3$ \\
\hline$T$ or $x^{2}$ & & 0.08 & 1.15 & & 1.70 & 0.29 & 0.98 & 1.40 \\
\hline$P$ & & 0.94 & 0.25 & & 0.25 & 0.77 & 0.33 & 0.16 \\
\hline
\end{tabular}


Table 2. SVC, AVC situation is related complications.

\begin{tabular}{|c|c|c|c|c|c|c|c|c|c|c|c|}
\hline \multirow[t]{2}{*}{ Puncture } & \multirow[t]{2}{*}{ Cases } & \multicolumn{2}{|c|}{ CRBSI } & \multicolumn{2}{|c|}{ Catheter blockage } & \multicolumn{2}{|c|}{ Catheter displacement } & \multicolumn{2}{|c|}{ Phlebitis } & \multicolumn{2}{|c|}{ Oozing } \\
\hline & & Cases & PCT $(\%)$ & Cases & $\operatorname{PCT}(\%)$ & Cases & $\operatorname{PCT}(\%)$ & Cases & $\operatorname{PCT}(\%)$ & Cases & $\operatorname{PCT}(\%)$ \\
\hline SVC & 61 & 3 & 4.4 & 6 & 9.8 & 11 & 18.0 & 10 & 16.4 & 8 & 13.1 \\
\hline AVC & 54 & 2 & 3.7 & 5 & 9.3 & 2 & 3.7 & 2 & 3.7 & 1 & 1.9 \\
\hline
\end{tabular}

